\title{
Combining front and back grating structures for broadband absorption enhancement in thin-film silicon solar cells
}

\author{
Aimi Abass ${ }^{1{ }^{*+}}$, Khai Q. Le ${ }^{2,3+}$, Peter Bienstman ${ }^{3}$, Andrea Alù ${ }^{2}$, Bjorn Maes ${ }^{3,4}$, and Marc Burgelman ${ }^{1}$ \\ ${ }^{I}$ Department of Electronics and Information Systems, Solar Cells Group, Ghent University, B-9000 \\ Ghent, Belgium \\ ${ }^{2}$ Department of Electrical and Computer Engineering, Metamaterials and Plasmonics Research \\ Group, The University of Texas at Austin, Austin, Texas, USA \\ ${ }^{3}$ Department of Information Technology, Photonics Research Group, Ghent University-imec, B-9000 \\ Ghent, Belgium \\ ${ }^{4}$ Department of Physics, Micro- and Nanophotonic Materials Group, University of Mons, B-7000 \\ Mons, Belgium \\ *Corresponding author: aimi.abass@elis.ugent.be \\ ${ }^{+}$Both Authors contribute equally to this work
}

\begin{abstract}
We investigate the possibilities of enhancing absorption in thin-film silicon solar cells with grating structures on the front ITO and back Ag contacts simultaneously. Broadband enhancement from complementary effects of each grating is demonstrated.
\end{abstract}

OCIS codes: (240.0040) Detectors : Detectors; (240.5350) Detectors : Photovoltaic; ( 240.0240) Optics at surfaces : Optics at surfaces; (240.6680) Optics at surfaces : Surface plasmons

\section{Introduction}

Driven by the increasing demand for clean energy, much research has been performed to develop low-cost and efficient solar cells (SCs). However, the use of low-cost silicon based materials for SCs tends to give rise to severe limitations in active material thickness, due to electrical reasons. For example in amorphous silicon (a-Si) SCs, due to high defect-related carrier recombination, the a-Si thickness is limited to a few hundred nanometers to keep the charge collection optimal. Similarly, in polycrystalline silicon (p-Si) SCs there is high carrier recombination at grain boundaries. This thickness limitation leaves the photon absorption to be inefficient when no proper light trapping techniques are utilized.

In the past few years, several techniques have been introduced to enhance absorption in thin film SCs [14]. One of the most widely used techniques is light-trapping caused by scattering surface textures [4]. This is done by tailoring the top contact of the cell, making it an efficient antireflection and scattering layer. Absorption enhancement has also been demonstrated by utilizing strong near field enhancement in plasmonic modes and by employing structures with resonantly enhanced scattering cross section [1-3].

In this work, we introduce triangular gratings both in the transparent conducting oxide (TCO) layer front contact and on the silver (Ag) back contact. We demonstrate a complementary absorption enhancement effect by the TCO grating in the shorter wavelength range via scattering and by the metal grating in the longer wavelength range via plasmonic mode excitation. Also, enhancement from cross interaction between the two gratings is observed.

Only results for amorphous silicon SCs are shown here, but the application of this double grating structure in polycrystalline silicon SCs will be investigated as well.

\section{Simulation Results}

The analysis is done using electromagnetic-wave simulations with COMSOL Multiphysics which employs the finite-element method. We model the light illumination on our structure as an incoming plane wave with wavelengths in the range 400-850 $\mathrm{nm}$. The sketch of the proposed device structure is depicted in Fig. 1(a). The TCO layer is made by indium tin oxide (ITO). The absorbing material is amorphous silicon (a-Si). The back contact is silver $(\mathrm{Ag})$. Triangular grating structures are introduced on the top ITO and bottom Ag layer. Here we show the optimal design for the case of single periodicity without any phase offset between the front and back contact gratings (the ITO triangles will always be directly on top of the Ag triangles). Fig. 1(b) shows the integrated absorption efficiency of the AM 1.5G spectrum in the 400-850 nm wavelength region 
for flat structures with different ITO and a-Si thickness. The oscillation seen is due to Fabry-Perot effects. We see that without any grating structure, even with a relatively thick a-Si layer of $820 \mathrm{~nm}$, we cannot obtain 90\% absorption efficiency. The thickness values indicated in Fig. 1(a) are the values for a planar SC structure which give a Fabry-Perot optimum absorption point with an electrically favorable a-Si thickness of 200nm.
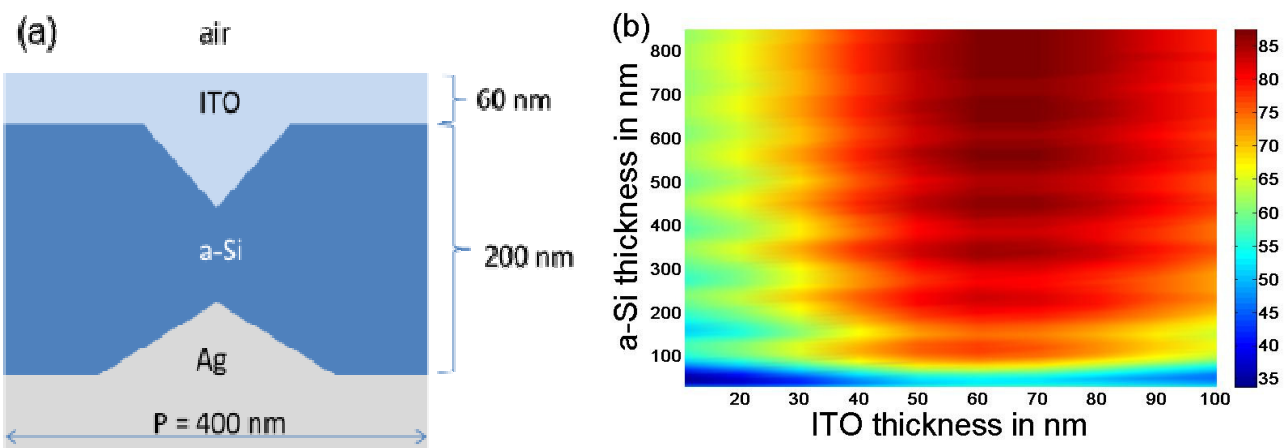

Figure 1. (a) The studied geometry. (b) Integrated absorption efficiency for flat structures in the $400-850 \mathrm{~nm}$ wavelength range.

In Fig. 2 we show several absorption spectra for different structures to demonstrate the enhancement provided by our double-grating structure. All the absorption spectra shown are for normal incidence case. The red line shows the absorption spectrum for an optimized flat cell. The peak we see at $725 \mathrm{~nm}$ wavelength for this flat case is due to a Fabry-Perot resonance. From this optimized flat structure, we introduce optimized triangular gratings on the ITO and Ag contacts with an optimized period of 400nm. For the Ag grating, the triangle height is $75 \mathrm{~nm}$ with a fill factor of 0.6 while, for the ITO grating, the triangle depth is also $75 \mathrm{~nm}$ with a fill factor of 0.4. The effect of using only the Ag back grating can be seen in the green curve in Fig. 2 . Additional surface plasmon polariton (SPP) and waveguide mode resonances arise in the 760-850 nm wavelength range, due to the periodicity on the metal surface, which enhances the absorption efficiency. The absorption performance for smaller wavelengths stays practically the same. By using only the front ITO grating structure (black curve), we see a broad enhancement nearly throughout the 400-720 nm wavelength region with only a relatively small weakening of absorption strength in the $720-770 \mathrm{~nm}$ wavelength region and little improvement beyond that. The ITO grating improves the absorption performance in the lower wavelength region by efficient light scattering.

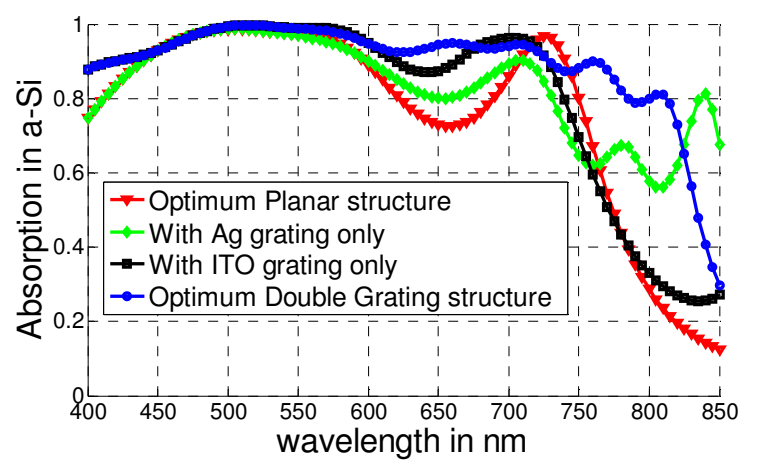

Figure 2. Absorption spectra of optimized SC structures for flat case (red line: with ITO thickness of $60 \mathrm{~nm}$ and a-Si thickness of 200 $\mathrm{nm}$ ), with ITO front grating only (black line: the structure is based on the same ITO and a-Si thickness with ITO grating depth of $75 \mathrm{~nm}$ and fill factor of 0.4), with $\mathrm{Ag}$ back grating only (green line: $\mathrm{Ag}$ grating height of $75 \mathrm{~nm}$ and fill factor of 0.6), and for double grating case (blue line).

Introducing both grating structures at once basically combines the best of both worlds, as it can be seen by inspecting the blue line in Fig. 2. It leads to a remarkable enhancement occurring nearly throughout the whole wavelength range of 400-850 $\mathrm{nm}$. Both gratings work together in a complementary manner. The back $\mathrm{Ag}$ grating again provides enhancement for the longer wavelength region while the ITO grating enhances the absorption performance at shorter wavelengths. $91.2 \%$ absorption of the AM 1.5G spectrum in the 400-850 $\mathrm{nm}$ wavelength region is achieved. This is $11.2 \%$ more compared to a flat cell with a thin a-Si thickness of $200 \mathrm{~nm}$ with optimized ITO thickness of $60 \mathrm{~nm}$.

There are four peaks of interest in the absorption spectrum for the combined grating structure, with maximum at wavelengths of $660 \mathrm{~nm}, 705 \mathrm{~nm}, 760 \mathrm{~nm}$, and $805 \mathrm{~nm}$. Fig. 3 shows the spatial absorption 
profile and the power propagation of the light in the structure. The spatial absorption profile at $600 \mathrm{~nm}$ and $660 \mathrm{~nm}$ wavelength shows how the ITO triangle scatters the light to increase absorption at shorter wavelengths. The ITO triangle first focuses the incoming light and then scatters it in the active material. The absorption peak at $660 \mathrm{~nm}$ is due to optimum interference effects in this scattering process. There is significant concentration of light absorption around the sharp metal back contact tip due to the lightning rod effect commonly seen in such sharp geometries. The spatial absorption profile at $705 \mathrm{~nm}$ shows a more distributed absorption throughout the active material, with maximum positions that are not directly between the bottom and top triangles. This indicates that the absorption peak is due to a standing-wave Fabry-Perot type mode. The spatial absorption profile at $760 \mathrm{~nm}$ and $805 \mathrm{~nm}$ wavelength is concentrated near the metal surface, but not only at the triangular metal tip, indicating that they are connected to SPP. While the peak at $805 \mathrm{~nm}$ is predominantly plasmonic, the peak at $760 \mathrm{~nm}$ is more mixed with a (standard) waveguide mode. This is seen from the field profile at those resonances (not shown) and from the pattern of the absorption in the active layer.

The two gratings are not entirely independent with respect to each other when both are present. We can see this from the red shifting of the waveguide and SPP resonance wavelengths. The red shift leads to more favorable resonance wavelength positions in this case. Intuitively, this red shift can be simply explained by noticing that the modes 'feel' a different effective refractive index due to the grating geometry. There is also interference between the modes, so a change in one will lead to a change in the wavelength position of both peaks.
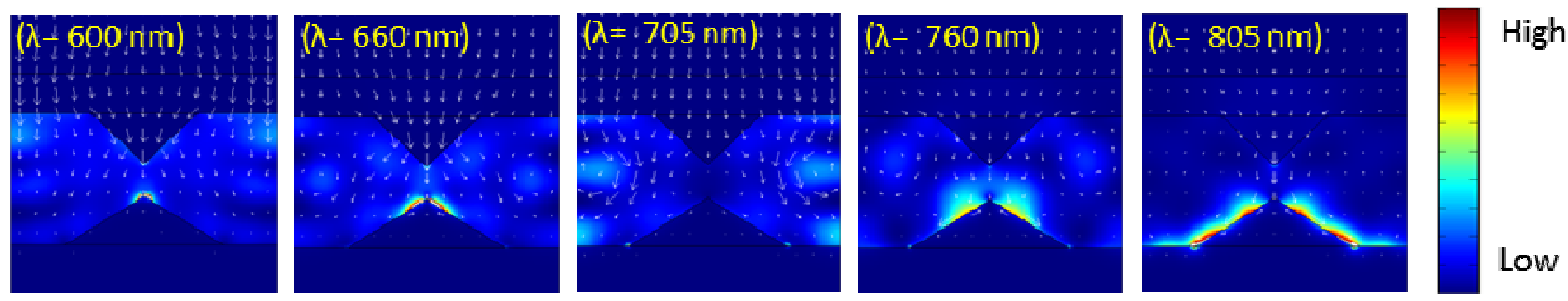

Figure 3. Absorption profiles at specific wavelengths (color scale: same scale in all graphs). The white arrows give the time averaged Poynting vector with normalized scale for each wavelength separately.

\section{Conclusions}

We show the possibility of achieving complementary absorption enhancement from simultaneous use of periodic gratings on the front and back contact of our cell. The ITO front contact grating is shown to enhance the shorter wavelength absorption performance through effective scattering of incoming light while the metal back contact grating enhances the longer wavelength regions via SPP and waveguide mode excitation. The combined effect of both grating structures leads to a broadband absorption enhancement in nearly the whole $400-850 \mathrm{~nm}$ wavelength range. The combined structure gives $91.2 \%$ absorption of the AM $1.5 \mathrm{G}$ spectrum which is $11.2 \%$ more compared to an optimized flat cell with acceptable a-Si thickness of $200 \mathrm{~nm}$.

\section{Conclusions}

This work is part of the Flemish IWT-SBO project SiLaSol (Number: 3E100243).

\section{References}

1. C. Hägglund and B. Kasemo, "Nanoparticle plasmonics for 2D photovoltaics: mechanisms, optimization and limits," Opt. Express 17, 11944-11957 (2009)

2. H. Shen, P. Bienstman, and B. Maes, "Plasmonic absorption enhancement in organic solar cells with thin active layers," J. Appl. Phys. 106, 073109 (2009).

3. A. Abass, H. Shen, P. Bienstman, and B. Maes, "Angle insensitive enhancement of organic solar cells using metallic gratings," J. Appl. Phys. 109, 023111 (2011).

4. R. Dewan, M. Marinkovic, R. Noriega, S. Phadke, A. Salleo, and D. Knipp, "Light trapping in thin-film silicon solar cells with submicron surface texture," Opt. Express 17, 23058-23065 (2009). 